\title{
High PHLPP expression is associated with better prognosis in patients with resected lung adenocarcinoma
}

Dongqing LV $v^{1,2}$, Haihua Yang ${ }^{1,3^{*}}$, Wei Wang ${ }^{1,3}$, Youyou Xie ${ }^{1,3}$, Wei Hu ${ }^{1,3}$, Minhua Ye $e^{4}$ and Xiaofeng Chen ${ }^{5}$

\begin{abstract}
Background: PH domain Leucine-rich-repeats protein phosphatase (PHLPP) is a novel family of Ser/Thr protein dephosphatases that play a critical role in maintaining the balance in cell signaling. PHLPP negatively regulates PI3K/Akt and RAF/RAS/' signaling activation, which is crucial in development, growth, and proliferation of lung cancer. The aim of this study was to investigate the association of PHLPP expression with biological behavior and prognosis of lung adenocarcinoma.
\end{abstract}

Methods: One hundred and fifty eight patients with pathologically documented stage I, II or IIIA lung adenocarcinoma were recruited in this study. Expression of PHLPP, p-AKT and p-ERK were evaluated by immunohistochemistry (IHC) in paraffin-embedded resected specimens. The correlation of their expression, which was dichotomized to low expression (a score of 0,1 ) versus high expression (a score of 2,3 ), with the clinicopathological parameters and prognosis of the patients also analyzed.

Results: High PHLPP expression rate in lung adenocarcinoma was $23.4 \%$. PHLPP expression level was significantly associated with tumor differentiation $(p=0.025)$ and tumor stage $(p=0.024)$. Patients with high expression of PHLPP showed significantly longer average survival time and higher 3 years survival rate than those with low expression of PHLPP (45 months versus 38 months, $85.8 \%$ versus $73.5 \%$ respectively) (Log rank test $x^{2}=7.086, p=0.008$ ). A significant inverse correlation was observed between PHLPP expression and p-AKT $(r=-0.523, p=0.000)$ or p-ERK $(r=-0.530, p=0.000)$.

Conclusion: Our results suggest that high levels of PHLPP might reflect a less aggressive lung adenocarcinoma phenotype and predict better survival in patients with lung adenocarcinoma. PHLPP can be a potential prognostic marker to screen patients for favorable prognoses.

Keywords: PHLPP, Lung cancer, Adenocarcinoma, Immunohistochemistry, Prognosis

\section{Background}

PHLPP (PH domain leucine-rich repeats protein phosphatase) represents a family of novel Ser/Thr protein phosphatases. PHLPP has been identified to negatively regulate PI3K/Akt and RAF/RAS/ERK signaling activation $[1,2]$. PI3K/Akt pathway plays a central role in inhibiting apoptosis in a variety of cell types including human cancer cells [3]. The RAF/RAS/ERK pathway plays a critical role

\footnotetext{
* Correspondence: yhh93181@hotmail.com

'Laboratory of Cellular and Molecular Radiation Oncology, Taizhou Hospital, Wenzhou Medical University, Zhejiang Province 317000, China

${ }^{3}$ Department of Radiation Oncology, Taizhou Hospital, Wenzhou Medical University, Zhejiang Province 317000, China

Full list of author information is available at the end of the article
}

in numerous cellular processes, including proliferation, differentiation, survival, and motility. Hyperactivation of RAF/RAS/ERK signaling is critical to the development of many human malignancies tumor $[2,4]$. The functional importance of PHLPP as a tumor suppressor in different types of cancer has been investigated in several recent studies $[1,2,5-13]$. The expressions of PHLPPs were frequently lost in a variety of human cancers, such as glioma [6], colon cancer [7, 8], prostate cancer [9], gastric cancer [10] and gallbladder cancer [11]. PHLPP expression was significantly associated with progression-free survival in gallbladder cancer [11]. The decrease in PHLPP1 level was highly correlated with shorter survival for patients with 
pancreatic ductal adenocarcinoma [12]. Patients with low PHLPP1 and PHLPP2 protein expressions have a poor prognosis and PHLPP1 was an independent prognostic factor in hypopharyngeal squamous cell carcinoma [13]. However, the expression and functional significance of PHLPP in lung adenocarcinoma are not clear. The present study aimed to investigate the association of PHLPP expression with biological behavior, clinicopathological characteristics and prognosis of lung adenocarcinoma.

\section{Methods}

\section{Patients}

Clinical data were compiled for 158 patients diagnosed with lung adenocarcinoma in resected specimen from 2008 to 2010 in Taizhou Hospital of Zhejiang Province. Variables included age, sex, date of diagnosis, stage at diagnosis, and time of follow-up. The follow-up was performed every 3 months after surgery for 2 years and once every 6 months thereafter. The median follow-up period was 38 months (range 3-56). Survival curves based on stage at time of diagnosis showed the expected patterns. All patients signed the informed consent. This study was approved by the Ethics Committee of Taizhou Hospital, and tissue specimen acquisition was carried out in accordance with institutional guidelines.

\section{Therapy}

All patients had an Eastern Cooperative Oncology Group (ECOG) perfor-mance status of 0 or 1, adequate baseline organ function defined as a leucocyte count $>$ $4 \times 109$ (absolute granulocyte count $>2 \times 109$, platelet count $>100 \times 109$, normal liver function tests and serum creatinine level $<1.4 \mathrm{mg} / \mathrm{dl}$ ) and no other severe comorbid conditions. Patient stage was redetermined according to the TNM Staging System of AJCC (7th version, 2009). Surgery was performed as the initial treatment for pathologically documented stage I, II and IIIA. Postoperative first-line chemotherapy was added depending on the TNM staging system. Cycles were repeated every 3 weeks and four cycles were delivered in II and IIIA patients.

\section{Immunohistochemistry}

At the tissue bank of Taizhou Hospital, tissue arrays were prepared using a Beecher manual arrayer. Fivemicrometre-thick sections of the paraffin-embedded tissue blocks were cut and mounted on polylysine coated slides. They were dewaxed in xylene and rehydrated through a graded series of ethanol. After deparaffinization, antigen retrieval treatment was performed at $120^{\circ} \mathrm{C}$ for $5 \mathrm{~min}$ in a $10 \mathrm{mM}$ sodium citrate buffer (pH 6.0). Endogenous peroxidase activity was blocked by using a $3 \%$ hydrogen peroxide solution at room temperature for $15 \mathrm{~min}$. Then, PHLPP (1:100, ab84978,
Abcam, Cambridge, England.), p-AKT (1:100, BS4007, Bioworld Technology, MN,USA.) and p-ERK (BS5016, 1:200, Bioworld Technology, MN,USA.) were applied and incubated overnight at $4^{\circ} \mathrm{C}$. After that, a thorough washing in a $0.01 \mathrm{M}$ phosphate-buffered saline (PBS) solution was done. The samples were then incubated with biotinlabeled goat anti-rabbit secondary antibody. Subsequently, binding sites of the primary antibody were visualized using a Dako EnVison kit (Dako, Glostrup, Denmark) according to the manufacturer's instructions. Finally, sections were counterstained with haematoxylin and mounted with glycerol gelatin. The immunohistochemical specificity of the antibodies was confirmed using two types of negative controls: (i) substituting rabbit non-immune IgG for the primary antibodies, and (ii) omitting the primary antibodies from the staining protocol. Normal colonic mucosa slide was used as a positive control.

\section{Staining evaluation}

Staining in tissues was evaluated by three pathologists who were blinded to any clinical details related to the patients. Membrane staining for PHLPP, cytoplasmic staining for $\mathrm{p}$-AKT and nucleus staining for $\mathrm{p}$-ERK were evaluated. The results of staining were scored according to the intensity of staining with Fourtier system (level 0 -3: negative $=0$, weakest $=1$, moderate $=2$, strong $=3$ ) [14]. Then, these scores were divided into PHLPP low expression group ( $0-1$ point) and PHLPP high expression group (2-3 point).

\section{Statistical analysis}

All statistical analyses were conducted using SPSS for Windows (version 17.0). Count data statistics using chisquare test. Associations between continuous variables were analyzed by Pearson's correlation test. Kaplan-Meier survival curves were calculated for the patient subgroups of interest and compared statistically, censoring for age and stage, using the log-rank test. A $p$-value $<0.05$ was considered statistically significant.

\section{Results}

A total of 158 patients with histologically proven lung adenocarcinoma were evaluated in this study. The expression of PHLPP, p-AKT and p-ERK in lung adenocarcinoma were evaluated by immunohistochemistry. The relationship between the expression of PHLPP and the clinicopathological characteristics of lung adenocarcinoma were analyzed. The results showed that the PHLPP expression was associated with histological differentiation and pathological $\mathrm{T}$ stage in lung adenocarcinoma (Table 1). Figure 1 showed the proportion of patients with different levels of PHLPP expression in lung adenocarcinoma. The structure of cases by the immunostaining scores of PHLPP expression highlights the relatively 
Table 1 Distribution of PHLPP expression in lung adenocarcinoma lesions according to clinicopathological parameters

\begin{tabular}{|c|c|c|c|c|c|c|}
\hline & \multirow{2}{*}{$\begin{array}{l}\text { No. } \\
\text { of } \\
\text { case }\end{array}$} & \multicolumn{4}{|c|}{ PHLPP expression } & \multirow[t]{2}{*}{$p^{*}$} \\
\hline & & 0 & 1 & 2 & 3 & \\
\hline \multicolumn{7}{|c|}{ Age (Median = 59 years) } \\
\hline$\leq 59$ years & 80 & 43 & 20 & 11 & 6 & \multirow[t]{2}{*}{0.515} \\
\hline$>59$ years & 78 & 34 & 24 & 11 & 9 & \\
\hline \multicolumn{7}{|l|}{ Gender } \\
\hline Male & 42 & 21 & 9 & 8 & 4 & \multirow[t]{2}{*}{0.357} \\
\hline Female & 116 & 56 & 35 & 14 & 11 & \\
\hline \multicolumn{7}{|l|}{ Differentiation } \\
\hline Well & 24 & 2 & 12 & 7 & 3 & \multirow[t]{3}{*}{0.025} \\
\hline Moderately & 93 & 46 & 25 & 12 & 10 & \\
\hline poorly & 41 & 29 & 7 & 3 & 2 & \\
\hline \multicolumn{7}{|l|}{ T stage } \\
\hline $\mathrm{T} 1$ & 25 & 12 & 3 & 6 & 4 & \multirow[t]{3}{*}{0.024} \\
\hline $\mathrm{T} 2$ & 97 & 43 & 38 & 14 & 2 & \\
\hline T3 & 36 & 22 & 3 & 2 & 9 & \\
\hline \multicolumn{7}{|l|}{ N stage } \\
\hline NO & 69 & 21 & 29 & 12 & 7 & \multirow[t]{3}{*}{0.322} \\
\hline N1 & 40 & 26 & 8 & 4 & 2 & \\
\hline N2 & 49 & 30 & 7 & 6 & 6 & \\
\hline \multicolumn{7}{|l|}{ Stage } \\
\hline I & 51 & 9 & 29 & 10 & 3 & \multirow[t]{3}{*}{0.896} \\
\hline ॥ & 51 & 31 & 9 & 6 & 5 & \\
\hline III & 56 & 37 & 6 & 6 & 7 & \\
\hline
\end{tabular}

${ }^{*} p$ is PHLPP difference between high and low expression. High expression is 2 and 3 , and lower expression is 0 and 1

low percentage $(23.4 \%)$ of cases with PHLPP, with score $2(13.9 \%)$ and $3(9.5 \%)$. Almost half $(48.7 \%)$ from all cases were shown to have no expression of PHLPP. Patients whose tumors stained at level 2 or greater were designated high expression. Patients whose tumors stained at level 1 or 0 were designated low expression.

Representative images of immunostaining of PHLPP, p-Akt and p-ERK expression in lung adenocarcinoma are shown in Fig. 2. There were $77.22 \%(122 / 158)$ of tumor tissues that had lost PHLPP expression. Among those with loss of PHLPP expression tumor tissues, the p-ERK positive rate was $63.11 \%(77 / 122)$, p-AKT positive rate was $62.30 \%$ (76/122), p-ERK and p-AKT both positive rate was $25.41 \%(31 / 122)$ (Table 2). A significant negative correlation was observed between PHLPP expression and p-AKT $(r=-0.523, P=0.000)$ or $\mathrm{p}$-ERK $(r=-0.530, P=0.000)$ (Table 2).

Among the 158 cases, there were 36 cases in which lung adenocarcinoma tissue demonstrated high expression with PHLPP antibody and 122 cases with low expression of PHLPP. Patients with high expression of

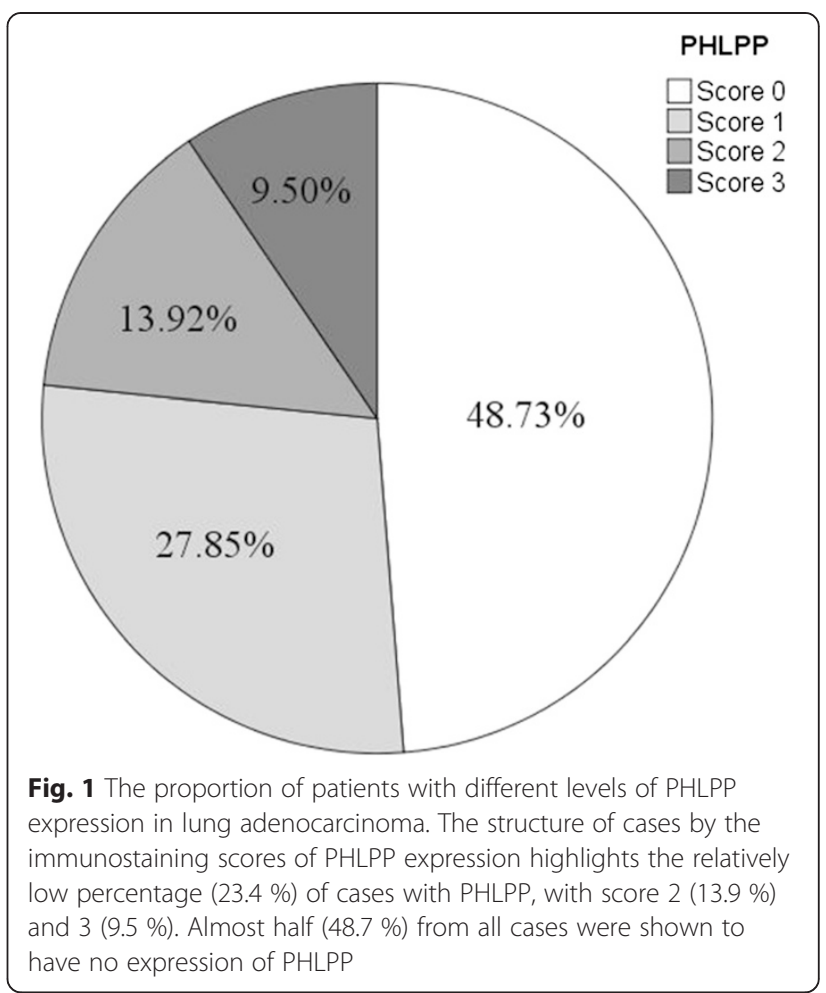

PHLPP showed significantly longer average survival time and higher 3 years survival rate than those with low expression of PHLPP (38 months versus 45 months, $73.5 \%$ versus $85.8 \%$ respectively) (Log rank test $\mathrm{X} 2=7.086, \mathrm{P}=0.008$ ) (Fig. 3 ).

There were no significant differences in OS among the PHLPP-negative \& p-AKT- positive, PHLPP-negative \& $\mathrm{p}$-ERK-positive and PHLPP-negative\& $\mathrm{p}-\mathrm{AKT} / \mathrm{p}$-ERKpositive ( $p=0.306$, Fig. 4$)$.

\section{Discussion}

Lung cancer is the most common malignant disease in the world, and is the leading cause of cancer morbidity and mortality in China, including both cities and countryside [15]. Strong evidence is emerging in the basic science literature that Akt and ERK are two signal transduction proteins that play important roles in carcinogenesis and chemoresistance [16]. PHLPP represents a family of novel Ser/Thr protein phosphatases that have been identified to negatively regulate signaling pathways activated including PI3K/Akt [1] and RAF/RAS/ERK in cancer cells [2]. Controlling the balance of protein phosphorylation is one of the most important defense mechanisms provided by protein phosphatases to prevent aberrant hyperactivation of signaling in cells [17]. Our studies here focused on elucidating the tumor suppressor function of protein phosphatases, PHLPP, in lung adenocarcinoma. In this study, we found that the expression of PHLPP were decreased in $76.5 \%$ of lung adenocarcinoma tissues, which is consistent 


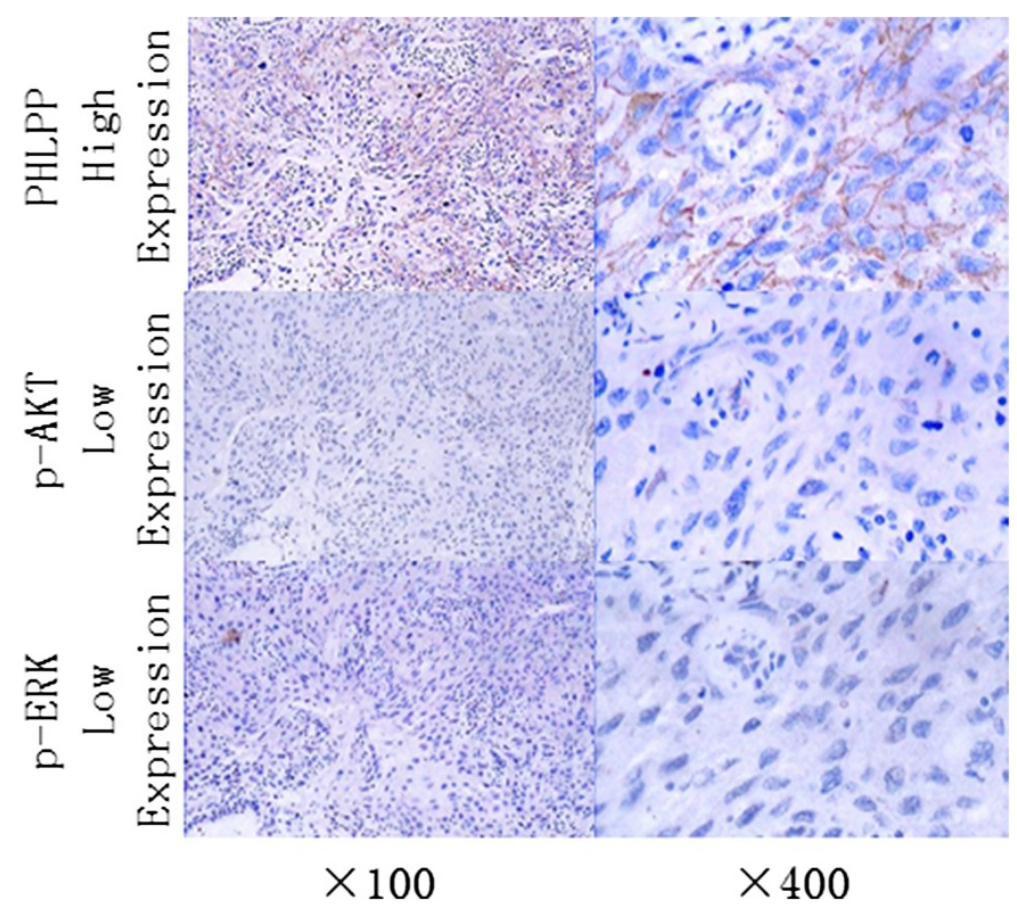

Fig. 2 Representative images of immunostaining of PHLPP, p-Akt and p-ERK expression in lung adenocarcinoma. The tissue sections from the lung adenocarcinoma were stained with the PHLPP (upper panels), p-AKT (middle panels) or p-ERK (lower panels) antibodies. The entire section was assessed at low (100x) (left panels) and high (400x) power (right panels) magnification

with previously findings in colon cancer, prostate cancer, chronic lymphocytic leukemia. Additionally, we found PHLPP expression was significantly correlated with tumor differentiation and $\mathrm{T}$ stage in lung adenocarcinoma. The level of PHLPP1 expression was significant related to the tumor T stage, in hypopharyngeal squamous cell carcinoma as reported by Zhou et al.

AKT and ERK signaling pathways are two important signaling pathways in the lung cancer [18]. The two signaling pathways are also the downstream signaling molecules of epidermal growth factor receptor (EGFR) signaling, which is mainly related with tumor occurrence and development. They also play an important role in the tolerance of chemoradiotherapy in lung cancer [19]. A few studies which assessed the role of Akt phosphorylation in NSCLC demonstrated that there was a statistically significant difference in survival between $\mathrm{p}$-Akt-positive and $\mathrm{p}$-Akt-negative patients

Table 2 The correlation between PHLPP, p-AKT, p-ERK for lung adenocarcinoma

\begin{tabular}{llcc}
\hline & & Pearson coefficient $(\gamma)$ & $p$ value \\
\hline p-AKT & PHLPP & -0.52 & 0.000 \\
p-ERK & PHLPP & -0.53 & 0.000 \\
p-AKT & p-ERK & -0.15 & 0.055 \\
\hline
\end{tabular}

and this difference was independent of tumor stage [20]. Activation of the ERK1/2 pathway is involved in malignant transformation both in vitro and in vivo. And the detection of immunoreactivity for p-ERK in patients with NSCLC is associated with advanced and aggressive tumors [21]. These data also suggest that the analysis of ERK1/2 activation may be useful to identify a subgroup of patients with a poorer prognosis. In the current study, we found PHLPP was inverse correlated with the expression of p-Akt and/or p- ERK in human lung adenocarcinoma tissues. Our findings here are consistent with our previous reports that PHLPP negatively regulated signaling pathways activated including PI3K/Akt and RAF/RAS/ERK in different cancer cells $[1,2]$. Moreover, in this study, for the first time we detected PHLPP expressions in human lung adenocarcinoma. Furthermore, we found that low expression of PHLPP in lung adenocarcinoma highly correlated with shorter survival, which is consistent with the recent report that [22] downregulation of PHLPP expression contributed to hypoxiainduced resistance to chemotherapy in colon cancer cells. This may be related with the differences of treatment outcomes. Wang et al. reported that the OS time and relapse-free survival (RFS) time in PHLPP1-positive patients were significantly longer than in PHLPP1 negative patients and PHLPP1 was 


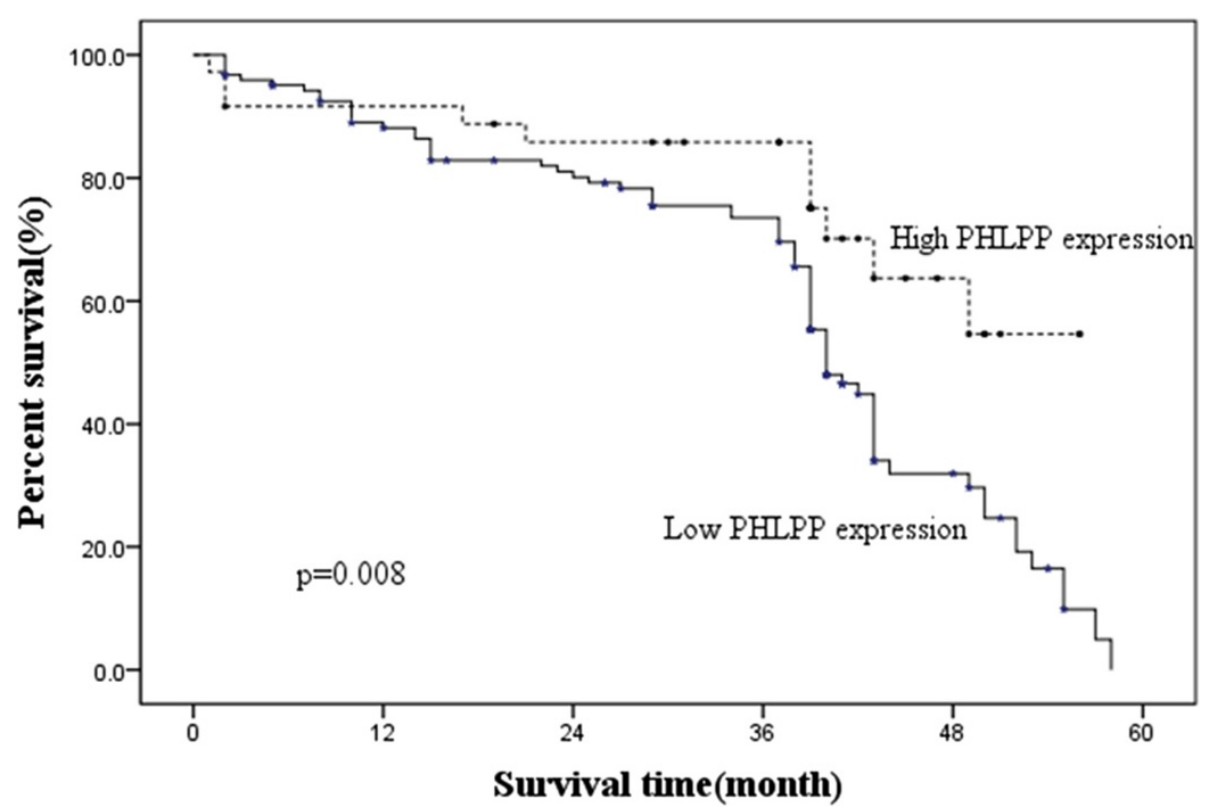

Fig. 3 Kaplan-Meier survival curves of patients with high and low expression of PHLPP. Patients with high expression of PHLPP showed significantly longer average survival time and higher 3 years survival rate than those with low expression of PHLPP (Log rank test $\left.x^{2}=7.086, P=0.008\right)$

an independent prognostic factor for OS and RFS of gastric cancer patient. Therefore, PHLPP may play an important role in the dual function of two signal pathways and would be better to inhibit the growth of tumor.

\section{Conclusions}

In conclusion, our study suggests that high levels of PHLPP might reflect a less aggressive lung adenocarcinoma phenotype and predict better survival in patients with lung adenocarcinoma. PHLPP can be a potential

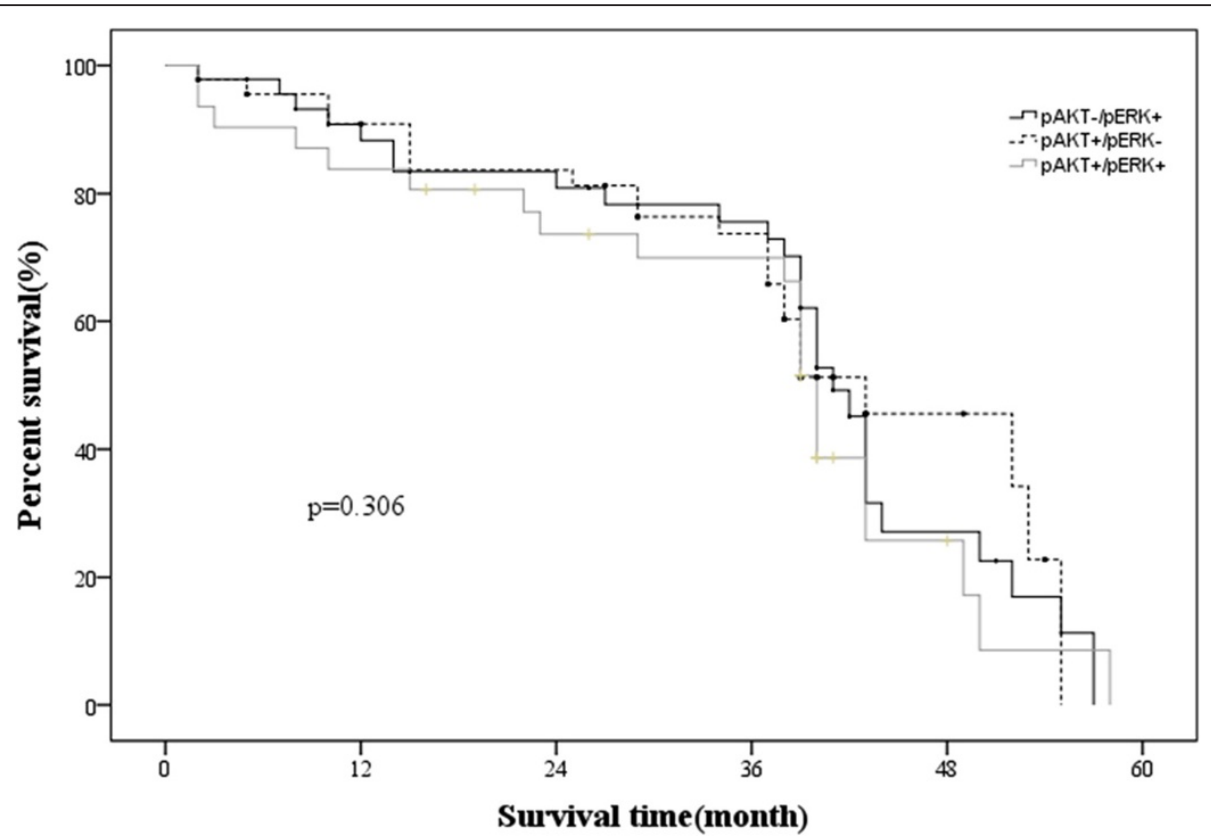

Fig. 4 Comparison of Kaplan-Meier survival curves for patients with different p-AKT and p-ERK expression levels in patients with PHLPP low expression. They are no differences in survival regardless of the expression of $p$-AKT and p-ERK in patients with low expression of PHLPP $(P=0.306)$ 
prognostic marker to screen patients for favorable prognoses.

\author{
Abbreviations \\ PHLPP: PH domain leucine-rich repeats protein phosphatase; \\ IHC: Immunohistochemistry; ECOG: Eastern Cooperative Oncology Group; \\ PBS: Phosphate-buffered saline; SPSS: Statistical package for the social \\ science; AJCC: American Joint Committee on Cancer; OS: Overall survival; \\ RFS: Relapse-free survival; EGFR: Epidermal growth factor receptor.
}

\section{Competing interests}

The authors declare that they have no competing interests.

\section{Authors' contributions}

DQ LV, HH Yang, W Wang and XF Chen Participated in the design and case selection of this study. YY Xie, W Hu and MH Ye performed the experiments. $\mathrm{HH}$ Yang and W Wang performed the data analysis. $\mathrm{HH}$ Yang also edited the manuscript while DQ LV, YY Xie, W Hu and MH Ye and XF Chen drafted the manuscript. All authors read and approved the final manuscript.

\section{Acknowledgements}

This study was supported by Zhejiang Provincial Medicine and Health Foundation (2013KYA225 and 2015KYA240) and Science and Technology Agency of Taizhou City (121KY08), China.

\section{Author details}

${ }^{1}$ Laboratory of Cellular and Molecular Radiation Oncology, Taizhou Hospital, Wenzhou Medical University, Zhejiang Province 317000, China. ${ }^{2}$ Department of Pulmonary Medicine, Taizhou Hospital, Wenzhou Medical University, Zhejiang Province 317000, China. ${ }^{3}$ Department of Radiation Oncology, Taizhou Hospital, Wenzhou Medical University, Zhejiang Province 317000, China. ${ }^{4}$ Department of Thoracic Surgery, Taizhou Hospital, Wenzhou Medical University, Zhejiang Province 317000, China. ${ }^{5}$ Enze Medical Research Center, Taizhou Hospital, Wenzhou Medical University, Zhejiang Province 317000, China.

Received: 23 February 2015 Accepted: 8 October 2015

Published online: 13 October 2015

\section{References}

1. Gao T, Furnari F, Newton AC. PHLPP: a phosphatase that directly dephosphorylates Akt, promotes apoptosis, and suppresses tumor growth. Mol Cell. 2005;18(1):13-24.

2. $\quad$ Li X, Stevens PD, Liu J, Yang H, Wang W, Wang C, et al. PHLPP is a negative regulator of RAF1, which reduces colorectal cancer cell motility and prevents tumor progression in mice. Gastroenterology. 2014;146(5):1301-12.

3. Brognard J, Clark AS, Ni Y, Dennis PA. Akt/protein kinase B is constitutively active in non-small cell lung cancer cells and promotes cellular survival and resistance to chemotherapy and radiation. Cancer Res. 2001;61(10):3986-97.

4. Brognard J, Dennis PA. Variable apoptotic response of NSCLC cells to inhibition of the MEKJERK pathway by small molecules or dominant negative mutants. Cell Death Differ. 2002;9(9):893-904.

5. O'Hayre M, Niederst M, Fecteau JF, Nquyen VM, Kipps TJ, Messmer D, et al. Mechanisms and consequences of the loss of PHLPP1 phosphatase in chronic lymphocytic leukemia (CLL). Leukemia. 2012:26(7):1689-92.

6. Molina JR, Agarwal NK, Morales FC, Hayashi Y, Aldape KD, Cote G, et al. PTEN, NHERF1 and PHLPP form a tumor suppressor network that is disabled in glioblastoma. Oncogene. 2012;31(10):1264-74.

7. Liu J, Weiss HL, Rychahou P, Jackson LN, Evers BM, Gao T. Loss of PHLPP expression in colon cancer: role in proliferation and tumorigenesis. Oncogene. 2009;28(7):994-1004.

8. Li X, Stevens PD, Yang H, Gulhati P, Wang W, Evers BM, et al. The deubiquitination enzyme USP46 functions as a tumor suppressor by controlling PHLPP-dependent attenuation of Akt signaling in colon cancer Oncogene. 2013;32(4):471-8.

9. Chen M, Pratt CP, Zeeman ME, Schultz N, Taylor BS, O'Neill A, et al. Identification of PHLPP1 as a tumor suppressor reveals the role of feedback activation in PTEN-mutant prostate cancer progression. Cancer Cell. 2011;20(2):173-86.
10. Wang Z, Shu H, Wang Z, Li G, Cui J, Wu H, et al. Loss expression of PHLPP1 correlates with lymph node metastasis and exhibits a poor prognosis in patients with gastric cancer. J Surg Oncol. 2013;108(7):427-32.

11. Qiu Y, Li X, Yi B, Zheng J, Peng Z, Zhang Z, et al. Protein phosphatase PHLPP induces cell apoptosis and exerts anticancer activity by inhibiting Survivin phosphorylation and nuclear export in gallbladder cancer. Oncotarget. 2015;6(22):19148-62.

12. Nitsche C, Edderkaoui M, Moore RM, Eibl G, Kasahara N, Treger J, et al. The phosphatase PHLPP1 regulates Akt2, promotes pancreatic cancer cell death, and inhibits tumor formation. Gastroenterology. 2012;142(2):377-87.

13. Zhou J, Yu X, Wang J, Li T, Jin T, Lei D, et al. Aberrant Expression of PHLPP1 and PHLPP2 Correlates with Poor Prognosis in Patients with Hypopharyngeal Squamous Cell Carcinoma. Plos ONE. 2015;10(3):e119405.

14. Reiner A, Neumeister B, Spona J, Reiner G, Schemper M, Jakesz R. Immunocytochemical localization of estrogen and progesterone receptor and prognosis in human primary breast cancer. Cancer Res. 1990;50(21):7057-61.

15. Siegel R, Ma J, Zou Z, Jemal A. Cancer statistics, 2014. CA Cancer J Clin. 2014;64(1):9-29.

16. McCubrey JA, Steelman LS, Abrams SL, Bertrand FE, Ludwig DE, Basecke J, et al. Targeting survival cascades induced by activation of Ras/Raf/MEK/ERK, PI3K/PTEN/Akt/mTOR and Jak/STAT pathways for effective leukemia therapy. Leukemia. 2008:22(4):708-22.

17. Brognard J, Newton AC. PHLiPPing the switch on Akt and protein kinase C signaling. Trends Endocrinol Metab. 2008;19(6):223-30.

18. Yajima I, Kumasaka MY, Thang ND, Goto Y, Takeda K, Yamanoshita O, et al. RAS/RAF/MEK/ERK and PI3K/PTEN/AKT Signaling in Malignant Melanoma Progression and Therapy. Dermatol Res Pract. 2012;2012:354191.

19. Katso R, Okkenhaug K, Ahmadi K, White S, Timms J, Waterfield MD. Cellular function of phosphoinositide 3-kinases: implications for development, homeostasis, and cancer. Annu Rev Cell Dev Biol. 2001;17:615-75.

20. David O, Jett J, LeBeau H, Dy G, Hughes J, Friedman M, et al. Phospho-Akt overexpression in non-small cell lung cancer confers significant stage-independent survival disadvantage. Clin Cancer Res. 2004;10(20):6865-71

21. Vicent S, Lopez-Picazo JM, Toledo G, Lozano MD, Torre W, Garcia-Corchon C, et al. ERK1/2 is activated in non-small-cell lung cancer and associated with advanced tumours. Br J Cancer. 2004:90(5):1047-52.

22. Wen YA, Stevens PD, Gasser ML, Andrei R, Gao T. Downregulation of PHLPP expression contributes to hypoxia-induced resistance to chemotherapy in colon cancer cells. Mol Cell Biol. 2013;33(22):4594-605.

\section{Submit your next manuscript to BioMed Central and take full advantage of:}

- Convenient online submission

- Thorough peer review

- No space constraints or color figure charges

- Immediate publication on acceptance

- Inclusion in PubMed, CAS, Scopus and Google Scholar

- Research which is freely available for redistribution 